\title{
CAMBIO DE VALORES EN LA POBLACION SUIZA
}

\section{OBSERVACIONES PRELIMINARES}

Cambio cultural y cambio social son puntos de referencia dentro de la teoría del cambio. El primero se refiere al cambio de los sistemas simbólicos; el segundo, al de las estructuras sociales. En el presente artículo nos centraremos en la cuestión del cambio de valores, pero debemos recordar que éste no es más que uno de los aspectos del cambio cultural en su conjunto.

Para definir el término valor me remitiré a la definición de Louis Guttman (Guttman/Levy, 1976):

Un tema determinado pertenece al universo de los valores únicamente si su campo de acción reclama una valoración cognoscitiva de la importancia de un/una $\left\{\begin{array}{l}\text { (objetivo) } \\ \text { (conducta) }\end{array}\right.$ con un carácter $\left\{\begin{array}{l}\text { (cognoscitivo) } \\ \text { (afectivo) } \\ \text { (instrumental) }\end{array}\right.$ en la esfera de la vida $(y)$ con un fin $\left\{\begin{array}{l}\text { (en sí mismo) } \\ \text { (más fundamental) }\end{array}\right.$ en la

\section{RềS}


esfera de la vida $(z)$, ordenándose jerárquicamente desde

(que fuese muy importante)

a

(que no lo fuese).

En comparación con las otras definiciones de que disponemos, ésta parece la más correcta y fecunda, pues:

- Corresponde a los patrones de definición dentro de una comprensión teórica de orientación estrictamente científica. Los elementos definitorios e hipotéticos no están mezclados en ella, como ocurre con frecuencia cuando se aplica el concepto de valor en Sociología y Psicología social. En la definición no se encierran elementos desconocidos, en el sentido de elementos ambiguos, ni tampoco una indefinida o vagamente definida serie de elementos.

- Frente a las definiciones contrarias, que conciben los valores como patrones internalizados para la elección de objetos y modos de comportarse, preferimos esta definición por abarcar mucho más, por lo que respecta a su contenido ya que el ámbito de su objeto se descompone en todas sus dimensiones, que aparecen con su denominación. Los conceptos teóricos y las hipótesis pueden ser formulados mejor como correspondencias entre un sistema definitorio y uno o más aspectos de la estructura empírica.

- Tiene en cuenta explícitamente el conocimiento deducible del estado actual de conocimientos sobre los valores, según el cual éstos se refieran ante todo a ámbitos vitales específicos en relación con los cuales hay que operar, no presuponiendo que sean patrones de preferencia omnicomprensivos, como hace Rokeach (1973).

Por lo demás, es inequívoco que los valores —como hace la inmensa mayoría de la moderna literatura sociológica y psicológica- se atribuyan aquí a personas y no a objetos, al contrario de lo que hacía la anterior doctrina de ciencias sociales y de economía política. A partir de este sistema definitorio es muy difícil, evidentemente, trazar una división lógica entre valores y actitudes.

Las actitudes se pueden definir así:

Un tema determinado pertenece al universo de las actitudes sólo si su ámbito reclama una conducta en relación con un objeto

(cognoscitivo)

de un modo (afectivo)

(instrumental)

y se ordena jerárquicamente en una escala (que va de muy positivo a muy negativo) con respecto a ese objeto. 
Esto significa que un valor es un caso especial de actitudes. La hipótesis plausible de una relación asimétrica entre valores y actitudes, sobre la base de una diferencia fundamental, como se supone normalmente, si bien no está bien fundamentada en la doctrina moderna - en la que los valores se colocan por delante de las actitudes y tienen funciones de dirección- ya ha sido abandonada. El problema de la relación mucho más compleja entre los distintos tipos de actitudes - al igual que los distintos tipos de conducta en generaltampoco se ha resuelto, encomendándose su solución a la investigación empírica.

La investigación sistemática del cambio de valores en las sociedades modernas ha sido iniciada recientemente por distintos equipos de investigadores (v. g.: Rescher, 1969; Meadows, 1972; Rokeach, 1974; Kmieciak, 1976), habiendo experimentado un aumento ${ }^{1}$. Las investigaciones teóricas (v. g.: Bell, 1973, 1973) y los estudios fundamentados empíricamente (véase Inglehart, 1977) sobre el tema coinciden con la creciente respuesta no sólo procedente de las ciencias sociales. Por otra parte, ello tiene relación con el hecho de que cada vez seamos más conscientes de los límites impuestos a la investigación de los indicadores objetivos $\mathrm{y}$, por tanto, del cambio estructural aislado. Por lo demás, cada vez resulta más evidente el carácter que poseen los valores de ser indicadores indispensables para el análisis de la conexión entre el sistema cultural y el social transmitida por la vía de la personalidad. A estos dos aspectos teóricos se debe añadir que en la moderna sociedad la relación entre el sistema cultural y el social se percibe cada vez de un modo más confuso. Algunas veces se habla de la desestabilización de la sociedad (Klages, 1975), de la transición a la sociedad postindustrial (Bell, 1973) o postmaterialista (Inglehart, 1977) o bien de la erosión del sistema de valores burgués (Rescher, 1969 y Noelle-Neumann, 1975).

Así, pues, los principales problemas que se plantean a la investigación de los valores son:

- La dirección del cambio de los valores en general y sus agentes.

- Las razones y los factores aceleradores de este cambio.

- Las diferencias de valores entre los grupos sociales.

- Las divergencias entre el cambio de los valores y el cambio social y, por ende, el problema de la legitimación de las estructuras sociales.

1 A este respecto me gustaría señalar las investigaciones de dimensión internacional e interdisciplinar, como el estudio realizado en ocho paises por Inglehart y otros sobre la protesta, la insatisfacción y el cambio en las sociedades postindustriales, 1974; la cooperación metódica, teórica y empírica de los investigadores de al menos nueve países, por hoy, en el subcomité de Indicadores de problemas sociales y problemas de valores de la Fundación Europea para la Ciencia, desde 1976; el proyecto mundial de indicadores, en el marco de la universidad de la ONU, y el primer simposio sobre actitudes y cambios de valores organizado por la Geselschaft für Zukunftsfragen de Berlín junto con la Gesamthochschule de Paderborn en mayo de 1978, al que asistieron más de cien investigadores de la República Federal de Alemania, Austria y Suiza. 
El proyecto financiero por el Fondo Nacional Suizo para la Promoción de la Ciencia y la Investigación, algunos de cuyos resultados examinaremos en los dos siguientes capítulos, trata de la población adulta suiza. Suiza presenta algunas peculiaridades que la convierten en un interesante objeto de investigación. Por un lado, es un ejemplo de una sociedad altamente industrializada; por otro, presenta el aspecto de un sistema muy tradicionalista $y$ especialmente estable. Ello se debe, en parte, a su tradición secular de democracia directa y a su peculiar modo de relacionar los derechos y los deberes civiles que se halla en el subsistema militar, así como en la existencia de múltiples lenguas. La investigación se basa en las siguientes cuestiones:

- ¿Qué valores y pautas de valor son dominantes en la población suiza?

- ¿Qué diferencias existen en las pautas de valor de cada uno de los subgrupos (Suiza alemana, francesa, italiana), los grupos de edad, los sexos, las clases económicas y niveles educativos, el campo y la ciudad?

- ¿Existen una conformidad y una elasticidad de valor entre las pautas de valor en los distintos ámbitos de la vida?

- ¿Dónde surgen problemas de legitimación?

- ¿Existen coincidencias o bien diferencias entre Suiza y otros países muy industrializados?

De acuerdo con Guttman, el modelo de investigación se puede describir en forma de esquema, como sigue:

Frase teóricamente diseñada para el estudio de los valores y los sistemas de valores en la población isuiza

El grado en que el entrevistado (x)

A

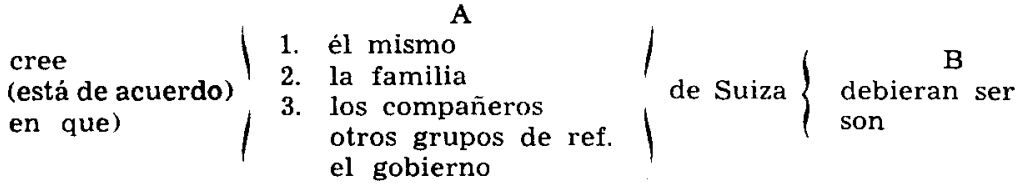

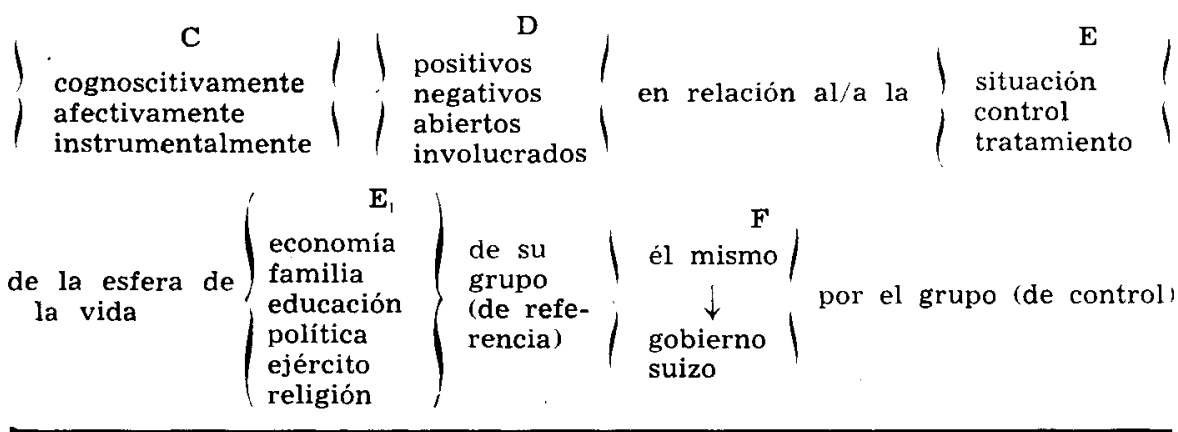




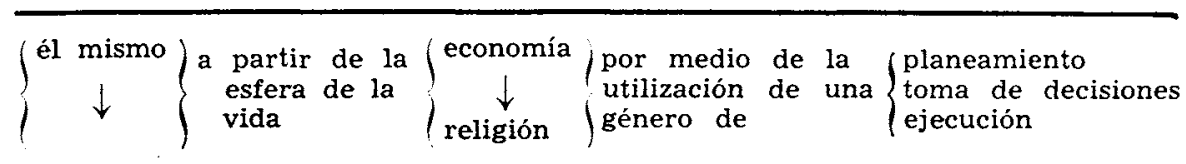

orientado temporalmente hacia el $\begin{aligned} & \mathbf{K} \\ & \text { pasado } \\ & \text { presente } \\ & \text { futuro próximo } \\ & \text { futuro remoto }\end{aligned} \mid$

orientado espacialmente hacia $\begin{aligned} & \text { la vecindad } \\ & \text { la ciudad } \\ & \text { la región nacional } \\ & \text { el conjunto del país } \\ & \text { lo internacional }\end{aligned} \mid$

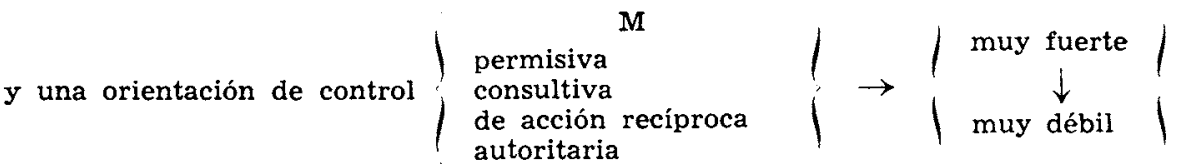

en el sentido de los elementos de las facetas de B a D.

Como se puede deducir de las cuestiones objeto de investigación, ésta se relaciona con un análisis de la situación real. Los datos fueron recogidos en 1976. Con los medios de que disponemos, por tanto, sólo podemos tratar el problema del cambio de valores de un modo indirecto y solamente en sus tendencias aproximadas. Por supuesto, únicamente pueden hacerse afirmaciones directas sobre el cambio social basándose en una investigación sistemática que abarque varios años, como se ha hecho en los últimos doce años en Israel (ver Guttman, 1971). Solamente entonces se puede responder a la cuestión, aún hoy no resuelta, de la relación entre el cambio estructural y el cultural. Se presenta la comparación de grupos de distinta edad y educación, así como la referencia a datos de otros países. A continuación haremos algunas reflexiones con ayuda de los resultados relativos a valores educacionales y los de instituciones públicas, tales como el mundo de la política y el ejército.

\section{VALORES EDUCACIONALES}

El cambio en los valores educacionales puede ser considerado como un importante indicador del cambio en una sociedad, como lo pone de manifiesto la literatura especializada. En efecto, un número creciente de estudios se ocupa de los valores como tema central de análisis (ver el General Survey, de NORC, Chicago, Allardt y otros, 1972; Ballestedt-Glatzer, 1975; Kmieciak, 1976; Bargel, 1978). 
En tales estudios, el papel socializante de la educación de los niños se considera básico (Hurrelman, 1976). En el esquema de Hurrelman el entorno social determina la naturaleza de la educación, refiriéndose a todos aquellos implicados en la educación como partícipes suministradores de referencia. Ellos suministran una perspectiva del tiempo presente y del pasado. Los valores educacionales son el resultante de estas referencias externas, a las que vuelven a referirse por vía del proceso interno de educación. Podemos considerar a estas personas de referencia como transmisores culturales: transmiten un juicio sobre lo que la sociedad considera importante.

Este concepto de socialización queda complementado por las teorías de la sociología del conocimiento. En ellas se supone que las pautas de orientación del juicio se establecen y son simbólicamente transmitidas en la educación, a través de la formación selectiva, siendo elaboradas selectiva y eficientemente las interpretaciones de la realidad (Berger-Luckman, 1966) y adquiriéndose destrezas afectivas e instrumentales que se convierten en acción directa. Las configuraciones de valor desempeñan un papel fundamental en las preferencias de la orientación del juicio y en el aspecto cognoscitivo de la acción directa. Con respecto al establecimiento de un juicio temporal - tanto en términos del propio establecimiento como en términos de su capacidad de previsión - la socialización debe ser siempre fluida y abierta al cambio. Podemos observar la legitimación percibida de las estructuras sociales en las pautas de orientación del juicio en general y en los valores educacionales en particular. Sin embargo, la falta o pérdida de legitimación, tan típica de las sociedades modernas, debe ser considerada también como una fuente esencial del cambio de valores y del correspondiente cambio social. Por ello, podemos suponer que la presión para el cambio, resultado de la socialización, repercutirá sobre el entorno social exterior.

La investigación de los valores educacionales es importante, además, porque la educación - por delante de otros temas - se ha convertido en el centro de un acalorado debate público. El hecho de que éste haya trascendido a los medios de comunicación social o de que las pruebas científicas aportadas en diversas polémicas sean a menudo inexactas o falsas, sólo sirve para añadir leña al fuego y darle más publicidad al tema (Bronfenbrenner, 1961; Lüscher, 1975; Inglehart, 1977). El efecto que producen los medios de comunicación social es ambiguo (Klapper, 1960), mas se puede asumir que acelera el cambio de valores y el consiguiente cambio social (Weiss, 1972).

\section{Hipótesis}

Al observar los valores educacionales de las modernas sociedades podemos partir de las siguientes hipótesis:

1. La importancia y significado de los valores educacionales varían de 
unos a otros grupos sociales, que se diferencian por el modo en que interpretan sus distintos entornos (Kohn, 1969; Wright-Wright, 1976; Heinz, 1976; Kmieciak, 1976; Bargel, 1978).

1.1. Las clases bajas siguen con algún retraso a las clases altas en considerar como importantes los valores educacionales (Bronfenbrenner, 1961; Kmieciak, 1976).

1.2. En distintos grupos sociales - pero especialmente en la clase media- existe una gran incertidumbre acerca de las líneas maestras y valores de la educación. Ello es patente en las incongruencias existentes entre los valores aducacionales particulares y las pautas generales de la educación (Irle, 1976; Kmieciak, 1976; Bergel, 1978).

2. Los valores de conformidad pierden importancia (Bronfenbrenner, 1961; Kmieciak, 1976; Bergel, 1978).

3. La autorrealización cobra importancia como valor (Bronfenbrenner, 1961; Lüscher, 1975; Kmieciak, 1976).

Los supuestos sobre los valores educacionales sólo pueden ser formulados y examinados de acuerdo con la dirección que parecen tomar, dados los conocimientos actuales. En este terreno no se han determinado aún tendencias a largo plazo que permitan establecer conclusiones más exactas.

\section{Valores educacionales en la población suiza}

Nuestros datos proceden de un estudio localizado y por ello reflejan fundamentalmente la situación actual en Suiza. Los cambios de valor sólo se pueden inferir indirectamente, comparando los grupos de edad y nivel educativo (ver infra), así como los datos de otros países.

La pregunta relevante de nuestra investigación contiene las siguientes 12 variables, entre las que los entrevistados deben escoger las tres más importantes y las tres menos importantes a su juicio, en relación con la educación ${ }^{2}$.

${ }^{2}$ Este modo de preguntar se emplea a menudo en la investigación con el resultado de un gran ensanchamiento de los dos extremos de la escala elegida. Por ello se debe abandonar todo intento de comprender la estructura exacta de una pauta de valor aislada. Esto es válido tanto para la zona media como para los extremos, pues es imposible establecer un orden de prioridades. Para obtener un resultado más exacto sería necesario clasificar cada respuesta por orden de preferencia desde muy importante hasta nada importante. 
TABLA 1

Valores educacionales y su importancia en la población suiza (en porcentajes)

\begin{tabular}{|c|c|c|c|c|}
\hline & & 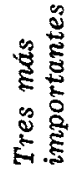 & 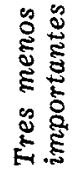 & 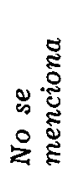 \\
\hline Tipo de variable ${ }^{3}$ & Variable & $(\%)$ & $(\%)$ & $(\%)$ \\
\hline Conformidad tradicional & $\begin{array}{llllll}\text { Obediencia } & \ldots & \ldots & \ldots & \ldots & \ldots \\
\text { Sentido del orden } & \ldots & \ldots & \ldots\end{array}$ & $\begin{array}{l}34 \\
18\end{array}$ & $\begin{array}{l}25 \\
18\end{array}$ & $\begin{array}{l}41 \\
65\end{array}$ \\
\hline 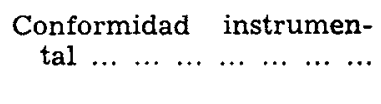 & $\begin{array}{l}\text { Capacidad de adaptación. } \\
\text { Gusto por el trabajo .. ... }\end{array}$ & $\begin{array}{l}17 \\
10\end{array}$ & $\begin{array}{l}17 \\
27\end{array}$ & $\begin{array}{l}66 \\
62\end{array}$ \\
\hline $\begin{array}{l}\text { Orientación a la eficien- } \\
\text { cia autónoma } \\
\ldots\end{array}$ & $\begin{array}{l}\text { Sentido de responsabili- } \\
\text { dad .................... } \\
\text { Apertura a nuevas ideas }\end{array}$ & $\begin{array}{r}55 \\
9\end{array}$ & $\begin{array}{r}4 \\
26\end{array}$ & $\begin{array}{l}41 \\
65\end{array}$ \\
\hline 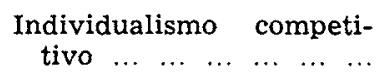 & Perseverancia $\ldots \ldots \ldots$ & 18 & 37 & 46 \\
\hline Autonomía crítica $\ldots \quad \ldots$ & $\begin{array}{ccccc}\text { Independencia } & \ldots & \ldots & \ldots & \ldots \\
\text { Autodisciplina } & \ldots & \ldots & \ldots & \ldots\end{array}$ & $\begin{array}{l}46 \\
20\end{array}$ & $\begin{array}{l}42 \\
17\end{array}$ & $\begin{array}{l}12 \\
63\end{array}$ \\
\hline Sociabilidad $\ldots \ldots \ldots \ldots$ & 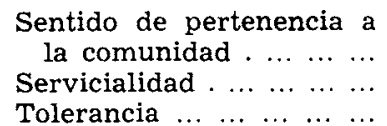 & $\begin{array}{l}20 \\
29 \\
21\end{array}$ & $\begin{array}{r}20 \\
9 \\
22\end{array}$ & $\begin{array}{l}61 \\
63 \\
57\end{array}$ \\
\hline
\end{tabular}

En esta tabla se puede observar, ante todo, que existen considerables diferencias entre la población con respecto a los valores educacionales. Sólo ha obtenido una mayoría el valor del sentido de responsabilidad, el cual - además del componente de eficiencia autónoma- también encierra el de sociabilidad. Entre determinados sectores de la población no está claro si hay que situarlo entre los tres valores más importantes de la escala o más abajo. En un estudio más detallado debería investigarse la hipótesis de que este valor se concentra en la zona media superior, siendo considerado por

${ }^{3}$ La manera de establecer las categorías corresponde aproximadamente a la de Bargel (1978). 
la población -incluso por el 41 por 100 que se abstiene- como básico en la percepción de los procesos sociales. El valor prosocial de servicialidad reúne una mayoría menor, pero aún importante. Son interesantes los valores gusto por el trabajo, perseverancia y apertura a nuevas ideas, que son considerados como importantes por un grupo relativamente pequeño y rechazados por una fuerte mayoría. Los resultantes - y controvertidos valorespresentan una gran dispersión en el conjunto medio.

Antes de entrar en el análisis pormenorizado, facilitaremos algunos datos procedentes de estudios comparables realizados en otros países, con el fin de valorar sus proporciones cuantitativas.

\section{TABLA 2}

Valores educacionales en poblaciones extranjeras en conjunto ${ }^{4}$ (los tres más importantes)

\begin{tabular}{|c|c|c|c|c|c|c|}
\hline $\begin{array}{lllllll}\text { Obediencia } & \ldots & \ldots & \ldots & \ldots & \ldots & \ldots\end{array}$ & 36 & 39 & 41 & 39 & 23 & 34 \\
\hline Gusto por el trabajo ... ... ... & - & - & $\ldots$ & - & 12 & 10 \\
\hline Apertura a nuevas ideas ..... & 27 & 33 & 36 & 43 & 19 & 9 \\
\hline Sentido de responsabilidad ... & - & - & 一 & - & 24 & 55 \\
\hline Autodisciplina $\ldots \ldots \ldots \ldots$ & 5 & 10 & 6 & 4 & 16 & 20 \\
\hline $\begin{array}{lllllll}\text { Servicialidad } & \ldots & \ldots & \ldots & \ldots & \ldots\end{array}$ & 29 & 16 & 24 & 19 & - & 29 \\
\hline
\end{tabular}

Según estos datos, Suiza es muy semejante a Dinamarca en lo que respecta al valor de conformidad de la obediencia y al valor prosocial - de la servicialidad. Suiza se halla muy distinta de los países escandinavos por lo que respecta al valor de la autodisciplina y a la apertura a nuevas ideas; al primero se le da más importancia; al segundo, mucha menos. Existen grandes diferencias entre todos los países europeos y los EE. UU., a excepción del valor de la autorrealización, tema en el que este último país coincide bas-

4 Todos los datos proceden del Scandinavian Welfare Survey de 1972 (Allardt, 1977) y del General Social Survey de NORC, Chicago, 1973. 
tante con Suiza. Como demuestran las otras respuestas a las variables, se podría hablar del énfasis que ponen los EE. UU. en los valores de autorrealización.

Sin embargo, no podemos comparar nuestro estudio con el conocido estudio EMNID de series temporales. Este estudio muestra el descenso de los valores de obediencia del 25 al 14 por 100 , así como una reducción del 41 al 37 por 100 en los valores del sentido del orden y diligencia. Ello contrasta con el aumento del valor de la independencia del 28 al 45 por 100 durante el período 1951-1972 (Ballerstedt-Glatzer, 1975). Los encuestados de la República Federal Alemana tenían que decidirse por una de tres respuestas dadas. No se puede deducir una pauta de valor de estas respuestas, pero sí una tendencia aproximada, comparando los tres valores.

Con el fin de evaluar algo mejor las tensiones y diferencias, nos gustaría investigar el material por grupos sociales. Para su clasificación elegiremos la combinación de variables sociodemográficas edad y educación. Una de las suposiciones que las ciencias sociales han heredado de la tradición doctrinal europea es la de que el cambio cultural y social ha sido básicamente obra de la juventud. Ahora bien, hay que ser cauteloso con esta presunción. En relación con este punto la literatura relevante considera evidente que se han extraído conclusiones a menudo prematuras sobre el cambio, despreciando los efectos del ciclo vital. Incluso hoy mismo sabemos muy poco de la relación entre ambos fenómenos. Sería necesario realizar investigaciones que cubriesen períodos de tiempo más largos. Sin embargo, hipotéticamente, de la relación de la curva de valores con los grupos de edades se podrían deducir al menos algunas conclusiones. Escogeremos los siguientes grupos de edades: veinteveintinueve años, como grupo que está iniciando su integración social por lo que respecta a la familia, profesión y vida pública; treinta-cincuenta y nueve años, como grupo que está plenamente integrado en la vida profesional y potencialmente también en la vida pública; y el grupo de más de sesenta años, como aquel que ya no desempeña roles sociales decisivos.

En los paradigmas sociocientíficos desarrollados por Deutsch (1961) y Lerner (1958) (véase también Etzioni, 1968; Lipset, 1972; Bell, 1973; Uüegg, 1973, 1978, e Inglehart, 1977), la educación juega un importante papel como base del cambio cultural y social. Su fundamento teórico se basa en la transición del lenguaje hablado al escrito como principal forma de comunicación. La elevación del nivel de educación académica lleva a la adquisición de nuevas destrezas cognoscitivas, así como a una transformación de las pautas informales de comunicación. Es la base de masivos cambios socioestructurales, como ocurre con el trasvase de mano de obra ocupada en la industria y agricultura a los servicios e industria del conocimiento (Bell, 1973). Ello varía fundamentalmente el sentido del entorno percibido por la población en su conjunto, siendo experimentado mucho más directamente por aquellos que están implicados en la producción y distribución de cono- 
cimientos. Independientemente de su posición o interpretación, todos los autores que se ocupan de este tema están de acuerdo en que estos procesos tienen que acelerarse. Los dos autores que más énfasis ponen en esta argumentación son Bell (1973) e Inglehart (1973).

Entre las variables externas, el nivel de educación académica ha demostrado poseer la máxima correlación estadística con los valores. Inglehart (1977) es explícito en este tema; de nuestros datos se puede llegar a la misma conclusión. Esto significa que sobre la base del nivel educativo son de esperar significativas diferencias entre los distintos grupos, pero no quiere decir que nosotros supongamos que exista concordancia dentro de un mismo nivel educativo. El mismo grado de educación académica puede dar lugar a distintas percepciones de juicio, como demuestran las anteriores consideraciones teóricas. Se puede comprender mejor nuestro esquema haciendo referencia a las variables sociodemográficas como elaboración auxiliar. Como han demostrado recientes investigaciones, el coeficiente de correlación entre tales valores es bastante bajo (véase Withey-Andrews, 1977). Valores de 0,20 a 0,30 con la variable educación son relativamente altos.

Para mayor claridad hemos dividido la variable de educación en sólo dos grupos: los que han abandonado los estudios al terminar la escuela elemental o media y aquellos que los han continuado posteriormente con plena dedicación. Las diferencias entre personas que han dejado la escuela a sus quince o dieciséis años, al terminar los estudios elementales o medios no son muy significativas. Los que sólo tienen estudios primarios subrayan los valores tradicionales - de conformidad- de obediencia y sentido del orden más que los otros. Este hecho pareció justificar que juntáramos ambos grupos. Si no diferenciamos entre los modos de educación superior y descartamos la categoría de una clase media educacional, las diferencias son insignificantes. Por tanto, no podemos diferenciar a los que producen y distribuyen conocimientos de los que los elaboran. Pero tenemos la ventaja de poder comparar grosso modo a las personas que están básicamente involucradas en el área del conocimiento con las que no lo están.

A partir de la diferencia entre, por un lado, la producción, distribución y la elaboración directa de conocimiento y, por otro, el consumo del mismo en el mejor de los casos, podemos deducir la hipótesis de que, en la percepción del grupo productor de conocimiento, los valores tradicionales de conformidad como la integración social son menos funcionales que para el grupo consumidor de conocimiento. Los resultados confirman rotundamente esta hipótesis (ver tabla 3). En los niveles más altos de educación, los valores de sentido del orden y obediencia, en comparación con todos los grupos de edad, se hallan clasificados con menos frecuencia entre los tres valores más importantes, lo que no deja de ser significativo. En comparación con los dos grupos de edad más jóvenes, es significativo que aquéllos se encuentren con menos frecuencia clasificados entre los tres menos importantes. En los niveles de 


\section{TABLA 3}

Valores educacionales según los grupos de educación y edad

\begin{tabular}{|c|c|c|c|c|c|c|}
\hline & \multicolumn{3}{|c|}{ Niveles de educación más bajos } & \multicolumn{3}{|c|}{ Niveles de educación más altos } \\
\hline & $\begin{array}{c}\text { Primer } \\
\text { grupo } \\
-\overline{-} \\
20-29 \text { años }\end{array}$ & $\begin{array}{c}\text { Segundo } \\
\text { grupo } \\
\overline{-} \\
30-59 \text { años }\end{array}$ & $\begin{array}{c}\text { Tercer } \\
\text { grupo } \\
\text { Más de } 60\end{array}$ & $\begin{array}{c}\text { Primer } \\
\text { grupo } \\
-\overline{-29 \text { años }}\end{array}$ & $\begin{array}{c}\text { Segundo } \\
\text { grupo } \\
- \\
\text { 30-59 años }\end{array}$ & $\begin{array}{c}\text { Tercer } \\
\text { grupo } \\
\text { Más de } 60\end{array}$ \\
\hline $\begin{array}{lllllllllllll}\text { Obediencia } & \ldots & \ldots & \ldots & \ldots & \ldots & \ldots & \ldots & \ldots & \ldots & \ldots & \ldots & \ldots\end{array}$ & $29: 26: 46$ & 33: $24: 43$ & 55: $13: 22$ & $10: 45: 45$ & $14: 43: 48$ & $34: 24: 42$ \\
\hline 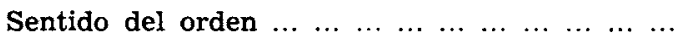 & $21: 23: 56$ & 19: $16: 66$ & 23: $10: 67$ & 2: $42: 56$ & $6: 28: 67$ & 21: $3: 76$ \\
\hline $\begin{array}{llllllll}\text { Capacidad de adaptación } & \ldots & \ldots & \ldots & \ldots & \ldots & \ldots & \ldots\end{array}$ & $21: 16: 62$ & $18: 15: 67$ & $12: 18: 70$ & $28: 20: 53$ & $18: 17: 65$ & 16: $18: 66$ \\
\hline 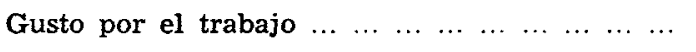 & $6: 30: 64$ & $11: 25: 64$ & $12: 22: 67$ & $6: 42: 53$ & $11: 36: 53$ & $7: 31: 63$ \\
\hline Sentido de responsabilidad $\ldots \ldots \ldots \ldots \ldots$ & 52: $8: 41$ & 55: $4: 41$ & 47: $5: 48$ & 62: $2: 36$ & $68: 3: 29$ & 50: $3: 47$ \\
\hline $\begin{array}{lllllllll}\text { Apertura a nuevas ideas } & \ldots & \ldots & \ldots & \ldots & \ldots & \ldots & \ldots\end{array}$ & $12: 26: 63$ & $8: 28: 65$ & $10: 30: 59$ & $11: 15: 74$ & $11: 19: 69$ & $5: 26: 69$ \\
\hline $\begin{array}{llllllllllll}\text { Perseverancia } & \ldots & \ldots & \ldots & \ldots & \ldots & \ldots & \ldots & \ldots & \ldots & \ldots & \ldots\end{array}$ & $18: 17: 45$ & $18: 38: 44$ & $14: 39: 47$ & $11: 26: 63$ & $25: 29: 46$ & $16: 48: 36$ \\
\hline $\begin{array}{lllllllllllll}\text { Independencia } & \ldots & \ldots & \ldots & \ldots & \ldots & \ldots & \ldots & \ldots & \ldots & \ldots & \ldots\end{array}$ & 56: $9: 34$ & 48: $12: 40$ & $36: 16: 48$ & 52: $7: 42$ & 14: $13: 44$ & $37: 15: 48$ \\
\hline $\begin{array}{llllllllllll}\text { Autodisciplina } & \ldots & \ldots & \ldots & \ldots & \ldots & \ldots & \ldots & \ldots & \ldots & \ldots & \ldots\end{array}$ & $17: 23: 61$ & $18: 19: 63$ & $17: 17: 62$ & $14: 28: 53$ & 26: $12: 62$ & 24: $7: 69$ \\
\hline Sentido de pertenencia a la comunidad ... ... & 23: $23: 55$ & $18: 21: 61$ & $15: 22: 63$ & $32: 12: 56$ & 23: 15:62 & $26: 13: 61$ \\
\hline $\begin{array}{llllllllllll}\text { Servicialidad } & \ldots & \ldots & \ldots & \ldots & \ldots & \ldots & \ldots & \ldots & \ldots & \ldots & \ldots\end{array}$ & 25: $7: 68$ & 29: $7: 64$ & 37: $8: 54$ & $13: 13: 74$ & $19: 14: 67$ & $8: 47: 45$ \\
\hline $\begin{array}{lllllllllllll}\text { Tolerancia } & \ldots & \ldots & \ldots & \ldots & \ldots & \ldots & \ldots & \ldots & \ldots & \ldots & \ldots & \ldots\end{array}$ & 18: $23: 58$ & $20: 22: 58$ & $13: 29: 58$ & 46: $9: 45$ & $31: 13: 57$ & $18: 24: 58$ \\
\hline
\end{tabular}

Primer número: Los tres más importantes.

Segundo número: Los tres menos importantes.

Tercer número: No se menciona.

Los niveles de educación más bajos se refieren a las personas que han abandonado los estudios después de la escuela elemental o media. cación.

Los niveles de educación más altos se refieren a las personas que han continuado después sus estudios con plena dedi- 
educación más bajos se observa un descenso en la clasificación positiva tanto del sentido del orden como de la obediencia. Pasado un lapso de tiempo, los grupos de nivel educativo bajo siguen la tendencia observada en los de nivel más alto. La clara ruptura existente entre las personas de mediana edad y edad avanzada $\left(2 .^{\circ}\right.$ y $3 .^{\circ}$ grupos de edad) en ambos niveles educativos nos permite suponer la existencia de un cambio, que también se observa en los resultados obtenidos en otros países.

En ambos niveles el valor de la capacidad de adaptación parece reflejar un fenómeno específico de la edad. El hacer mayor hincapié en el grupo de edad más joven en comparación con los otros dos grupos de edad, de cara a la mayor capacidad de integración requerida, hace plausible una percepción más elevada de la funcionalidad de este valor, al menos para una parte de esta cohorte de edad.

Ya hemos señalado la poca importancia atribuida al valor gusto por el trabajo en el resumen de los resultados (tabla 3 ). El porcentaje de. los que sitúan este valor entre los tres menos importantes aumenta entre los de edad avanzada. En comparación con los demás valores, alcanza su punto más alto entre los del nivel educativo más bajo. Entre los de nivel educativo más alto se sitúa a la misma altura que el rechazo de la conformidad tradicional. Este incremento puede explicarse por referencia a la época de la vida de los encuestados, pero es tan pequeño en realidad que se podría concluir que es un fenómeno de cambio. Sería interesante examinar cómo clasifican este valor las personas del 64 por 100 o el 53 por 100 que no mencionan en absoluto el gusto por el trabajo. Dado que la población suiza, como se observa en otros resultados, manifiesta un gran gusto por el trabajo, se puede extraer la hipótesis de que ese 64 ó 53 por 100 se concentran en la mitad superior. Valdría la pena investigar la hipótesis de Habermans (1973) de que por lo que respecta a la educación en la familia moderna, el gusto por el trabajo se considera ajeno al papel de las instituciones educativas. Si esta hipótesis fuese válida valdría la pena estudiar si conduce a un descenso del gusto por el trabajo. Asimismo, se debería investigar si la historia de la educación coincide en este punto.

En contra de la hipótesis de que los grupos de nivel educativo más alto están más orientados hacia el futuro, se demuestra que los distintos grupos sólo se diferencian en relación con el valor de apertura a nuevas ideas. La ruptura neta existente entre el segundo y el tercer grupo de edad apunta a un fenómeno de cambio, pudiéndose presumir que esta valoración va en aumento. Pero este cambio tiene lugar mucho más lentamente que en otros países (ver tabla 2). La autonomía, como queda manifestada en el valor de independencia, está clasificada en un lugar muy alto, concordante con la devaluación de la conformidad tradicional. Este valor sale ganando gracias a su correlativo, el sentido de responsabilidad, que llega a ser aún más importante entre los de nivel educativo alto que entre los de bajo nivel. 
Finalmente, los valores prosociales parecen ser muy importantes en la pauta de valoración de ambos grupos. De acuerdo con la hipótesis expresada en diversas teorías y deducible de nuestro precedente discurso teórico, es evidente que los grupos de educación más elevada subrayan más los valores universales y prosociales (sentido de pertenencia a la comunidad, tolerancia), mientras que los de nivel más bajo tienden a hacer hincapié en los valores particularistas (servicialidad). Pero la tendencia hacia una transferencia del valor de servicialidad al de sentido de pertenencia a la comunidad que se observa en el grupo de nivel más bajo, nos permite concluir que el horizonte de referencia se está ampliando como resultado del paso del tiempo.

Desafortunadamente, solamente podemos comparar indirectamente nuestros datos con los de una investigación semejante que se está realizando en la República Federal de Alemania (Barcel, 1978). En este país se observan las mismas tendencias que en Suiza. De la mayor diferenciación en las pautas valorativas de los distintos grupos educacionales podemos inferir que en Alemania existen más tensiones en los valores. Al mismo tiempo, la fuerte degradación de los valores de conformidad tradicional y la escasa importancia que se da en Suiza a los valores modernos, como es la apertura a nuevas ideas, indican la existencia de un cambio más lento pero más homogéneo.

Hemos intentado razonar la elección de las dos variables socio-demográficas (educación y edad) para el análisis de los valores educacionales. Sería interesante añadir que las diferencias entre los sexos son pequeñas. Las mujeres tienden a subrayar algo más la servicialidad y la capacidad de adaptación, al tiempo que otorgan una importancia ligeramente inferior a la autonomía (independencia). Se podría hablar de un fenómeno de cambio en la creciente semejanza de las opiniones de ambos sexos.

Al principio de este capítulo establecimos la hipótesis de que los grupos sociales se diferencian por el modo de interpretar distintos entornos en relación con sus valores educacionales. Nos gustaría examinar esta hipótesis aplicando un análisis del mínimo espacio, método desarrollado por Louis Guttman (Guttman, 1968). Por este método se puede determinar la distancia o proximidad de las variables diseñadas. También se pueden generar hipótesis sobre la estructura del valor espacio sin utilizar preguntas escalonadas. Como ejemplo tomaremos el grupo de mediana edad (treinta-cincuenta y nueve años) y los dos grupos educativos de que hemos tratado anteriormente. El gráfico $\mathrm{I}^{5}$ muestra los valores educativos del grupo de mediana edad dentro del nivel educativo inferior; el gráfico II $^{5}$, los valores educacionales del mismo grupo de edad dentro del nivel de educación superior.

Podemos afirmar lo siguiente:

- En contraste con los grupos de nivel educativo alto, en los de bajo nivel el sentido del orden está orientado hacia la eficiencia y tiene un aspecto claramente prosocial.

5 Nota 5 al final del artículo. 
- Los de bajo nivel perciben la capacidad de adaptación como un va. lor universal, mientras que los de nivel alto la consideran emparentada con el individualismo competitivo.

- El gusto por el trabajo y la independencia se encuentran unidos más frecuentemente en los grupos de bajo nivel que en los de alto.

- Para los grupos de nivel más alto el sentido de responsabilidad posee una faceta de autonomía crítica, mientras que en los de nivel más bajo este valor se ve orientado a la eficiencia.

- Para los de nivel inferior la apertura a nuevas ideas es una cualidad personalísima; en cambio, para los de educación superior se encuentra estrechamente relacionada con la capacidad de adaptación.

- Para los de educación inferior la autodisciplina posee claramente un componente de orientación a la eficiencia, en tanto que para los grupos de educación superior aquélla es significativamente prosocial.

- En opinión de los de educación superior, la independencia está claramente relacionada con la sociabilidad universalista, mientras que para los de educación inferior dicho valor tiene una connotación orientada a la eficiencia.

De este resumen se infiere evidentemente que entre los grupos de nivel educativo inferior prevalecen los aspectos orientados a la eficiencia, en tanto que en los grupos de nivel superior domina la orientación hacia la sociabilidad.

Resumiendo este capítulo podemos decir que las hipótesis $1,1.1$ y 2 han resistido la prueba. Pueden ser diferenciadas además especialmente respecto a la hipótesis 1 y 1.1 .

La hipótesis 3 parece estar necesitada de revisión, al menos por lo que respecta a nuestros datos (ver también Bargel, 1978). No existe un predominio de la autorrealización, incluso entre los grupos de nivel educativo más alto. En estos mismos los valores individualistas están claramente relacionados con los de una sociabilidad universalista. Por lo que atañe a la hipótesis 1.2 , sólo podemos decir que, gracias al análisis, las contradicciones tlegan a ser invisibles. Esta cuestión debe ser reexaminada por el análisis de los valores $\mathrm{y}$, especialmente, de los procesos de sección directa. Asimismo deberían examinarse los procesos históricos específicos de largo alcance en las sociedades respectivas.

\section{VALORES MANTENIDOS EN RELACION CON LA ESFERA PUBLICA}

Ahora, con la ayuda de ejemplos tomados de la esf́era pública, como son el mundo de la política y el de la milicia, estudiaremos si se descubren fe- 


\section{GRAFICO I}

Valores educacionales de los grupos con menor nivel educativo

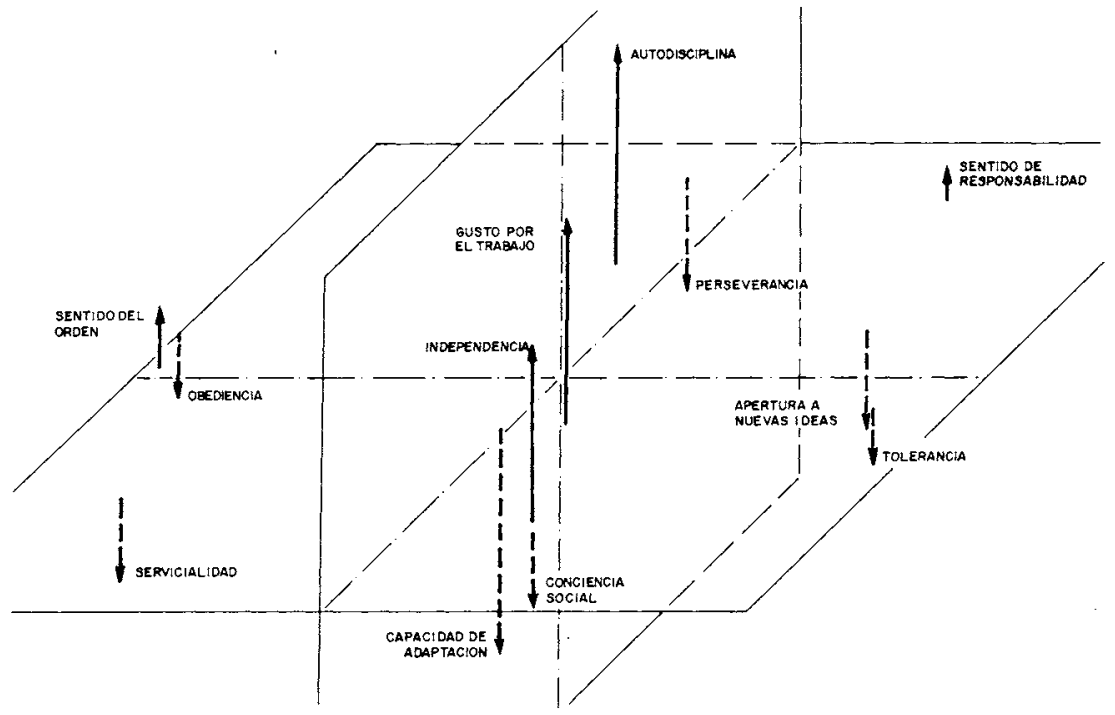

\section{GRAFICO II}

Valores educacionales de los grupos con mayor nivel educativo

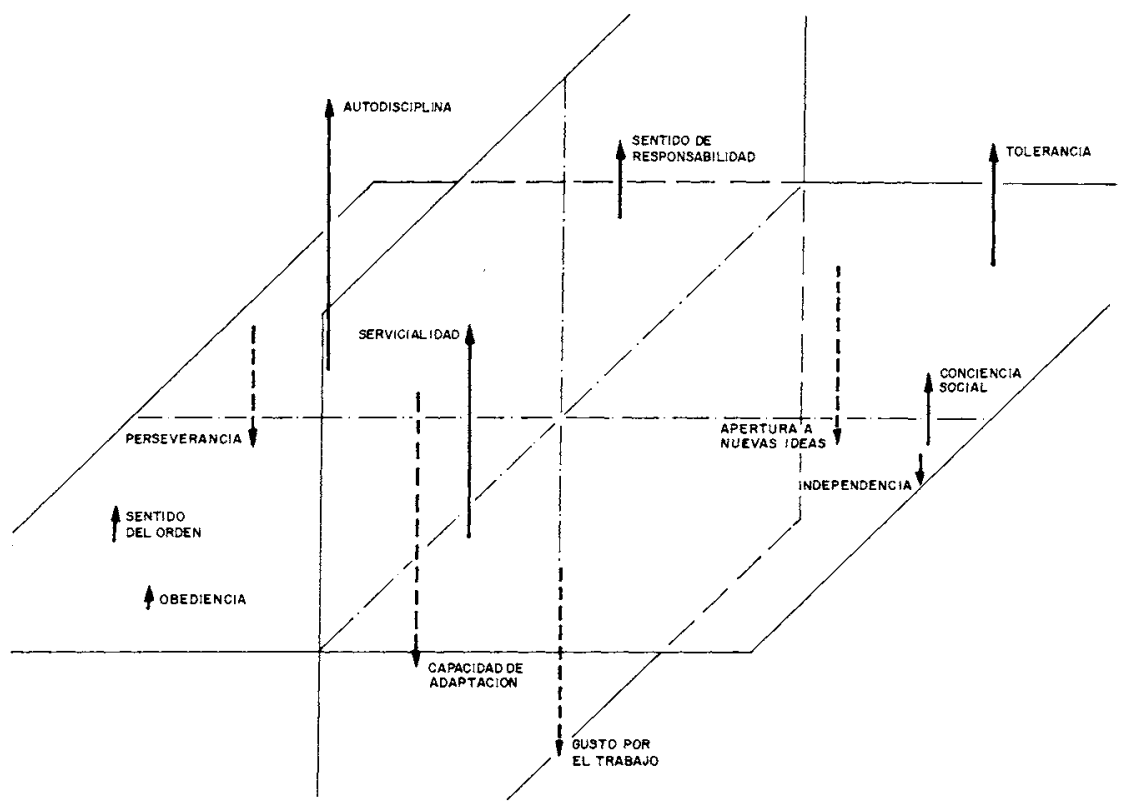


nómenos similares a los observados en la educación. La principal cuestión que se plantea es conocer la relación del ciudadano con las instituciones públicas. Así podremos estudiar la legitimación percibida de la estructura y de la actuación política.

El análisis de la valoración de la esfcra pública es importante en tanto en cuanto nos capacita para ver hasta qué punto repercuten en los valores y en el cambio de los mismos las instituciones profundamente enraizadas. En Suiza uno se puede preguntar si su centenaria democracia directa está sujeta a los mismos problemas de legitimación que en otros países. Diversos autores se han preguntado si Suiza podría resolver estos problemas de legitimación.

En nuestra investigación presentamos a los entrevistados dos preguntas paralelas: qué valores debían ser más destacados, en su opinión, y qué valores menos subrayados en relación con las esferas política y militar. Los rer sultados se presentan en la tabla $4^{6}$.

TABLA 4

Valores en la esfera política y militar

\begin{tabular}{|c|c|c|c|c|c|}
\hline \multirow{2}{*}{ Tipo de variable } & \multirow{2}{*}{ Variable } & \multicolumn{2}{|c|}{$\begin{array}{c}\text { Debe ser } \\
\text { más } \\
\text { subrayada }\end{array}$} & \multicolumn{2}{|c|}{$\begin{array}{c}\text { Debe ser } \\
\text { menos } \\
\text { subrayada }\end{array}$} \\
\hline & & $\begin{array}{l}\text { Pol. } \\
(\%)\end{array}$ & $\begin{array}{l}\text { Mil. } \\
(\%)\end{array}$ & $\begin{array}{l}\text { Pol. } \\
(\%)\end{array}$ & $\begin{array}{l}\text { Mil. } \\
(\%)\end{array}$ \\
\hline \multirow{4}{*}{$\begin{array}{llll}\text { Conformidad } & \ldots & \ldots & \ldots\end{array}$} & Obediencia a los superiores & 41 & 62 & 59 & 38 \\
\hline & Sentido del orden $\ldots \ldots \ldots$ & 80 & 79 & 20 & 21 \\
\hline & $\begin{array}{ccccccc}\text { Disciplina } & \ldots & \ldots & \ldots & \ldots & \ldots & \ldots\end{array}$ & 70 & 65 & 30 & 35 \\
\hline & Tradicionalismo $\ldots \ldots \ldots \ldots$ & 27 & 25 & 73 & 75 \\
\hline \multirow{3}{*}{$\begin{array}{l}\text { Orientación a la efi- } \\
\text { ciencia autónoma ... }\end{array}$} & $\begin{array}{llllll}\text { Patriotismo } & \ldots & \ldots & \ldots & \ldots & \ldots\end{array}$ & 40 & 51 & 60 & 49 \\
\hline & Sentido de responsabilidad. & 95 & 91 & 5 & 9 \\
\hline & $\begin{array}{ccccccc}\text { Tolerancia } & \ldots & \ldots & \ldots & \ldots & \ldots & \ldots\end{array}$ & 82 & 82 & 18 & 18 \\
\hline & Igual trato para todos . ... & 94 & 90 & 6 & 10 \\
\hline Sociabilidad & $\begin{array}{c}\text { Compañerismo } \\
\begin{array}{cccccc}\text { grupal) } & \ldots & \ldots & \ldots & \ldots & \ldots\end{array}\end{array}$ & 86 & 96 & 14 & 4 \\
\hline
\end{tabular}

- Aquí aparecen resumidos los resultados de las categorías de respuestas: debería ser subrayado más y debería ser subrayado mucho más, así como la de deberia ser subrayado menos y debería ser subrayado mucho menos. 
Por lo que se refiere a la esfera política es interesante anotar que los valores de conformidad fueron rechazados siempre. El valor de apego a la tradición fue rechazado con mucha fuerza ( 73 por 100 ), pero también lo fue el de patriotismo (60 por 100$)$, la identificación con símbolos nacionales del pasado. El sentido de la tradición es inexistente en la mayoría de la población suiza y es incierta la correspondiente legitimación de la actuación en el sistema político. La gran demanda de orden en términos cuantitativos (70 por 100) puede ser una respuesta a ello.

La importancia atribuida al orden es enorme, especialmente si se compara con los resultados obtenidos en otros países. Inglehart (1973) aporta los siguientes datos de los países del Mercado Común y los EE. UU.:

\section{TABLA 5}

El ordien como meta de las sociedades occidentales, 1973

(porcentaje de los que consideran este objetivo como el primero o segundo en importancia entre doce)

Mantener el orden

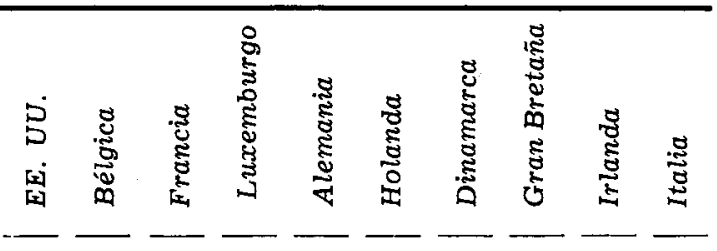

$\begin{array}{llllllllll}20 & 10 & 21 & 28 & 18 & 18 & 31 & 11 & 16 & 17\end{array}$

$(\%)(\%)(\%)(\%)(\%)(\%)(\%)(\%)(\%)(\%)$

Inglehart plantea preguntas sobre objetivos relativamente concretos, clasificando las respuestas según las dimensiones de valor: economía, seguridad, pertenencia, autorrealización.

Dejando aparte los distintos métodos de plantear las preguntas - los encuestados tenían que escoger dos de los doce objetivos nacionales propuestos, por lo que era de esperar una gran variedad y porcentajes bajos-, el análisis de los datos de Inglehart revela que, por término medio, en Europa los objetivos económicos descubiertos en los lemas lucha contra el aumento de precios, crecimiento económico, economía estable, y el objetivo de la seguridad, transfondo de la frase lucha contra el crimen, se clasifican por delante del mantenimiento del orden. En los EE. UU. el crecimiento económico viene inmediatamente después del mantenimiento del orden. Dado que todos estos objetivos son tendentes hacia el valor abstracto que representa el 
orden, podemos concluir que éste ocupa un lugar prioritario. Hay que señalar que lo que se preguntaba no era el orden propiamente, sino el sentido del orden. Como revela el análisis de Minissa, el sentido del orden está estrechamente relacionado con la disciplina. El criticismo de los ciudadanos respecto de la actuación política no se refiere tanto al Estado en abstracto, sino más bien a la actuación de los ciudadanos en el contexto de la democracia directa $\mathrm{y}$, en último término, al mismo ciudadano.

Los valores de orientación autónoma a la eficiencia y sociabilidad con. cuerdan con los valores de pertenencia en las categorías de Inglehart. Al contrario que en otros países, en Suiza se sitúan sin excepción por delante del valor de conformidad de orden. Respecto a la cuestión de la legitimación en la política suiza, es interesante observar que un tercio de las respuestas percibían que la tradición democrática había violado repetidas veces el principio de igualdad de trato. Señalaban que este valor debía ser subrayado mucho más, de donde resulta el máximo porcentaje obtenido por esta categoría.

Tanto en la esfera de lo militar como en la de la política se observa la misma tendencia. En la primera también la orientación hacia la eficiencia autónoma y la sociabilidad tienen preferencia sobre los otros, mientras que los valores de conformidad tradicional son los más dudosos. El principio de obediencia a la jerarquía militar recibe más apoyo que la obediencia a las personas situadas en posición superior en la esfera política, pero no hay que olvidar que el 38 por 100 cuestiona la tradicional y rígida jerarquía militar. Así, pues, el ejército suizo sufre problemas de legitimación (compárese con Harries-Jenkins/van Doorn para otros países). Lo mismo podemos decir del valor del patriotismo. Cuando aproximadamente el 50 por 100 de la población empieza a dudar de esta base de legitimación del ejército tradicional, hay que pensar que están cambiando las relaciones entre ejército y sociedad.

Con el fin de determinar las diferencias existentes entre la población, hemos analizado la jerarquía de los valores educacionales en los distintos niveles de educación (ver tabla 1 ). Volveremos a resumir los resultados obtenidos del modo siguiente:

- Disminución de la legitimación de la conformidad tradicional, proceso que está muy avanzado entre los grupos de alto nivel de educación y que va progresando, aunque con retraso entre los grupos de menor nivel.

- En general, el grupo de nivel educativo más alto hace hincapié en la orientación de eficiencia autónoma, que es un aspecto de la eficiencia instrumental, en tanto que para los de nivel más bajo es un aspecto de la sociabilidad universal.

- Importancia creciente de los valores prosociales entre los grupos de más alto nivel educativo.

- Desplazamiento de la sociabilidad particularista hacia otra de tipo universalista. 
¿En qué medida concuerdan estos resultados con los obtenidos respecto a las esferas política y militar? Entre los dos grupos existen las siguientes diferencias:

- Los grupos de nivel educativo menor hacen más hincapié en los valores de conformidad tradicional; a saber: apego a la tradición y pa. triotismo. Por lo que respecta a este último, ello sólo es válido para el ámbito de la política. En el terreno militar no existen marcadas diferencias. Los grupos que menos subrayan estos valores son los dos primeros (de menor edad).

- El primer grupo de edad (veinte-veintinueve años) es el que menos apoyo da al valor de conformidad de orden.

- Lo mismo se puede decir del valor de disciplina.

- Los grupos de más alto nivel educativo subrayan más el valor de conformidad de obediencia a personas de rango superior que las de nivel más bajo. El grupo más joven es el que lo rechaza con más fuerza.

- El valor de sentido de responsabilidad, generalmente aceptado, tiene más respaldo entre los grupos de nivel educativo más alto. Tiene incluso un componente prosocial.

- Lo mismo podemos decir de un valor de sociabilidad universalista como es la tolerancia.

- Lo contrario ocurre con el valor de compañerismo, de sociabilidad particularista.

- Aquí no aparece claramente una transferencia de la orientación particularista a la universalista, pero es cierto que ambos valores están clasificados generalmente muy altos.

- Por lo que respecta a la posición en la escala de la conformidad tradicional, los grupos de nivel educativo inferior siguen a regañadientes a los de nivel superior.

En las esferas política y militar se da una concordancia de valores y tendencias. Ello podría respaldar la hipótesis del cambio. Las diferencias de valoración del orden y la obediencia a las personas de rango superior pudiera estar basada en la distinta percepción de las estructuras. Podemos, ciertamente, adelantar la hipótesis de que la función de la socialización está relacionada con el cambio a largo plazo. Pero los resultados obtenidos no se pueden comparar directamente, dados los diferentes métodos aplicados en las encuestas. En cualquier caso existen diferencias, pues las estructuras de las distintas es. feras de la vida se perciben a través del prisma de la experiencia.

Con respecto a la cuestión de la educación no se estableció ningún valor comparable con el de igual trato para todos. Curiosamente este valor, generalmente muy bien clasificado, tiene más importancia para los de alto nivel educativo que para los de bajo. Un resultado semejante se obtuvo con respecto al valor de la obediencia a las personas de rango superior. $\mathrm{La}$ consir 
deración de las diferencias de los resultados en otros terrenos, especialmente en el de la economía, muestran una tendencia similar. Por ello hay que re currir, obviamente, a la teoría de la carencia relativa (Runciman, 1968; ver también Marsh, 1977), aunque algunos de sus elementos deban ser reconsiderados posteriormente. Parece que en Suiza la percepción de la carencia relativa está más relacionada con la apatía política que con la actitud de protesta (ver gráfico III).

La existencia expuesta de deficiencias o problemas de legitimación, tanto en el terreno político como en el militar, debe acarrear consecuencias en el apoyo que obtengan ambas instituciones. Quisiéramos examinar esta suposición a continuación:

El apoyo que obtienen los dos sistemas citados puede considerarse alto, teniendo en cuenta la extensión del rechazo real hacia ellos.

- Para el 11 por 100 de los que contestan, la democracia suiza es la mejor de todas las que existen actualmente;

- el 75 por 100 consideran la democracia suiza como aceptable;

- el 12 por 100 piensan que en esa democracia deben cambiarse muchas cosas para satisfacer sus expectativas;

- y el 2 por 100 opinan que no hay peor democracia que la suiza.

- El 49 por 100 están de acuerdo con la siguiente afirmación: el ejército juega un papel primordial en la vida pública suiza, cosa que es conveniente;

- el 45 por 100 califican al ejército de mal necesario.

- y el 6 por 100 juzgan que debería ser suprimido.

En conjunto, un 2 por 100 del total de la muestra de población critica fundamentalmente al subsistema político, mientras que un 6 por 100 critica fundamentalmente el subsistema militar. El rechazo total de la democracia suiza entre el 2 por 100 de los que contestan no depende de su edad ni de su educación. (Ver tabla 6.)

Ahora bien, la identificación acrítica con el sistema actual se presenta de manera muy distinta; en efecto, desciende significativamente a medida que disminuye la edad, cayendo del 23 por 100 al 6 por 100 en los grupos de menor nivel educativo y del 16 por 100 al 5 por 100 en los de más alto nivel. La absoluta confianza en Suiza no desciende sobre todo en el grupo de edad más corta, sino que la disminución es más evidente entre el grupo tercero y segundo.

De las dos categorías medias, que reflejan ya sea una actitud de crítica constructiva ya $s \in a$ una crítica radical, predomina decididamente la primera. Esta es la opinión de la mayoría de la población suiza, que refleja una relación bastante distante y abierta, pero fundamentalmente positiva con el Estado suizo, sobre cuya base se puede garantizar mejor el principio esencial 
de la democracia directa - el constante diálogo entre el ciudadano y el Estado- que sobre la base de una identificación total, enlazada afectivamente. Esta actitud instrumental conduce a la apatía política que hemos descubierto en el 30 por 100 de la población gracias a las preguntas posteriores.

La petición de reformas fundamentales depende de la edad, pero no de la educación. En el grupo más joven es una exigencia de casi la quinta parte, mientras que en los otros dos grupos de edad sólo lo es de la décima parte de los que contestan. La correlación de ésta con otras preguntas de la investigación demuestra que este duro criticismo es el resultante de una actitud fundamentalmente socialista. Pero también puede suponer formas de apatía política, como revela la descripción de Minissa. No podemos precisar si las diferencias entre los grupos de edad indican un cambio o son un fenómeno específico de la edad.

\section{TABLA 6}

Apoyo a la democracia suiza y al ejército segín el nivel de educación y el grupo de edad

\begin{tabular}{|c|c|c|c|c|c|c|}
\hline & \multicolumn{3}{|c|}{$\begin{array}{c}\text { Grupos de nivel } \\
\text { más bajo }\end{array}$} & \multicolumn{3}{|c|}{$\begin{array}{l}\text { Grupos de nivel } \\
\text { más alto }\end{array}$} \\
\hline & $\begin{array}{l}1 .{ }^{\circ} \\
(\%)\end{array}$ & $\begin{array}{l}2 \dot{\circ}^{\circ} \\
(\%)\end{array}$ & $\begin{array}{l}3.0^{\circ} \\
(\%)\end{array}$ & $\begin{array}{l}1 \% \\
(\%)\end{array}$ & $\begin{array}{l}2 .^{\circ} \\
(\%)\end{array}$ & $\begin{array}{l}3.0^{\circ} \\
(\%)\end{array}$ \\
\hline \multicolumn{7}{|l|}{ Democracia: } \\
\hline . La mejor democracia $\ldots . .$. & 6 & 9 & 23 & 5 & 7 & 16 \\
\hline Más o menos aceptable $\ldots$ & 75 & 78 & 63 & 75 & 82 & 76 \\
\hline Cambio radical $\ldots \ldots \ldots \ldots$ & 17 & 11 & 12 & 19 & 11 & 9 \\
\hline Muy mala $\ldots \ldots \ldots \ldots \ldots$ & 2 & 2 & 2 & 1 & 1 & - \\
\hline \multicolumn{7}{|l|}{ Ejército: } \\
\hline Papel fundamental..$\ldots \ldots$ & 38 & 51 & 66 & 21 & 35 & 46 \\
\hline 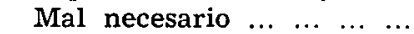 & 48 & 45 & 32 & 60 & 58 & 52 \\
\hline Debería ser suprimido .... & 14 & 5 & 2 & 19 & 7 & 2 \\
\hline
\end{tabular}

Primer grupo, 20-29 años; segundo grupo, 30-59 años; tercer grupo, más de 60 años.

Con respecto al papel de los militares en la sociedad suiza podemos abservar una tendencia semejante en lo fundamental, tal y como he demostrado en mi trabajo, en este congreso, para el seminario Inter-Universidad sobre las Fuerzas Armadas. Comparando los grupos de edad descubrimos un desplazamiento aún más importante desde la identificación incuestionada con la institución militar a una actitud positiva y distante, pero básicamente instrumental, en ambos grupos de nivel educativo. Los grupos de nivel más bajo parecen seguir la tendencia de los de nivel más alto. Es significativa la proporción de opiniones negativas registradas en los dos grupos de edad más jóvenes. 
Estos resultados constituyen un desafío a la legitimidad de la institución, debiendo ser considerados como innovadores. Hasta en Suiza la nación y el ejército parecen ser ya menos importantes para la identidad emocional del individuo moderno. La integración del mismo en la sociedad y en su subsistema directo, el ejército, es cada vez más resultado de factores cognoscitivos e instrumentales. Mas la discrepancia del individuo es resultante de presuponer la existencia de unos ciudadanos informados, críticos y, por consiguiente, emancipados y de una democracia capaz de innovación.

Antes de terminar este capítulo demostraremos mediante la descripción de Minissa las correlaciones de la muestra de población. Hemos añadido algunas variables más del proyecto con el fin de completar el cuadro. (Gráfico III: Valores y apoyo al sistema político y militar) ${ }^{7}$.

\section{OBSERVACIONES FINALES}

A través de nuestro análisis de las tendencias del cambio en la población suiza hemos llegado a las siguientes conclusiones:

- Los valores universalistas y prosociales tienden significativamente a ocupar un lugar central en las pautas de valor; son correlativos con los valores de autonomía individualista.

- Los valores de eficiencia competitiva son periféricos.

- Cada vez se ponen más en duda los valores de conformidad tradicional. Por tanto, éstos también se desplazan hacia la periferia.

- Los agentes del cambio de valores son los grupos de nivel educativo más alto.

- Existen tensiones latentes de considerable magnitud que quedan expresadas por la distinta importancia jerárquica que atribuyen los distintos grupos educacionales a los mismos valores; pero los grupos de nivel educativo más bajo siguen la tendencia de los de nivel más alto. Por ello apenas existen clases en relación con los valores.

- Este cambio de valores lleva a un problema de legitimación de las estructuras sociales. Para el ciudadano, las instituciones públicas adquieren un carácter crecientemente instrumental.

En consecuencia, el cambio de valores de la población suiza muestra importantes paralelismos con el cambio observado en otras sociedades altamente industrializadas. Parece resultado de aquellas tendencias al cambio que no tienen ninguna relación con la erosión de los valores burgueses o con la revolución silenciosa, sino que van unidas a la difusión de los valores civiles como la libertad, la igualdad y la fraternidad entre toda la población; valores

${ }^{7}$ Nota 7 al final del artículo. 


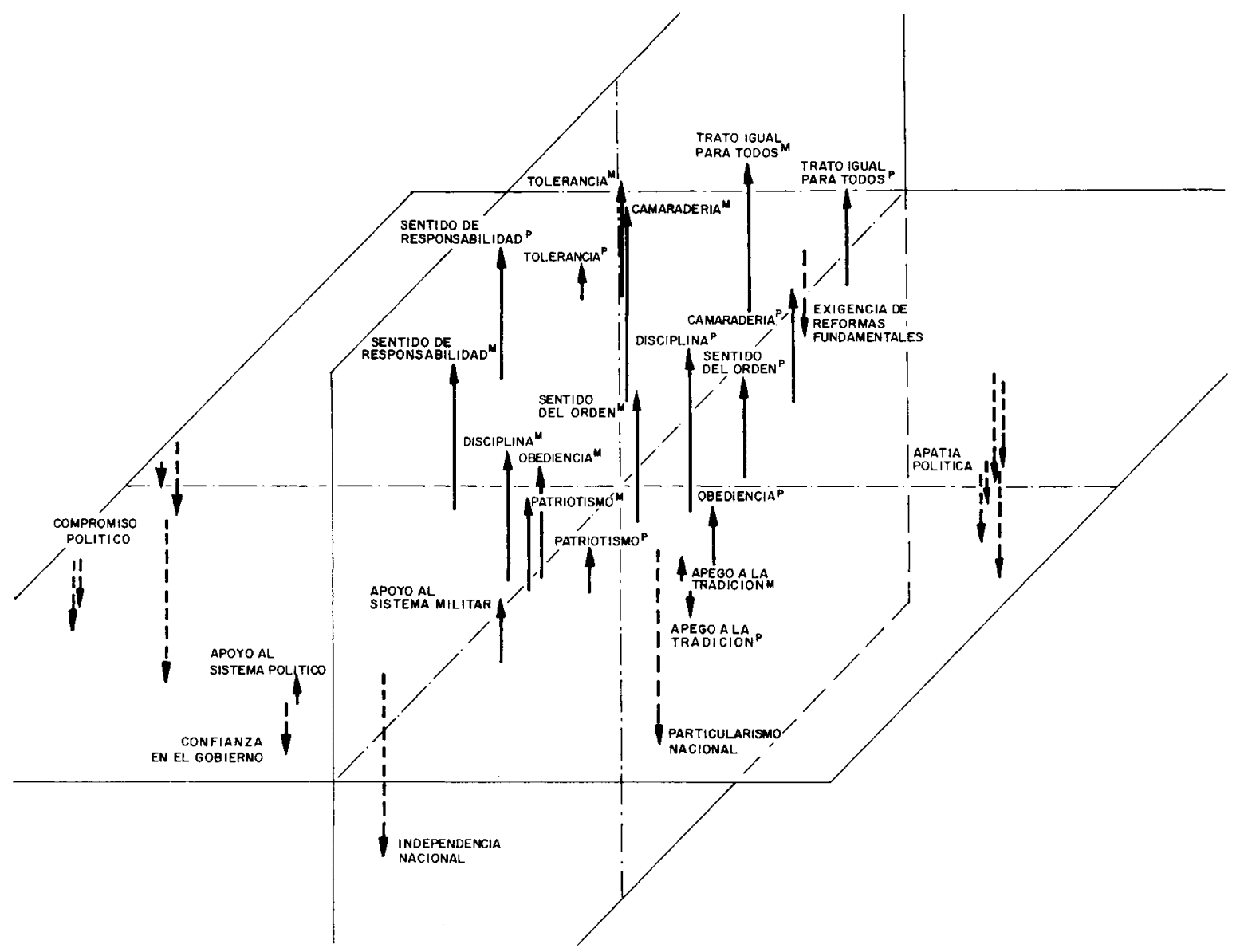


por los que lucharon los líderes de la burguesía en la Revolución francesa en contra del orden feudal. En las estructuras sociales que conservan algún elemento preburgués se plantean numerosos problemas de legitimación, derivados del proceso de transferencia de poder a la burguesía. Este cambio de valores es síntoma de un proceso básico característico del proceso de modernización de la sociedad occidental. El modo en que éste tiene lugar y los problemas específicos que plantea vienen determinados por la tradición histórica de cada sociedad. Muchos factores parecen indicar que el proceso de cambio en la sociedad suiza, comparado con el de otras sociedades, se efectúa de un modo relativamente suave, cosa que puede explicarse por la estabilidad y profundo enraizamiento de la tradición democrática en Suiza. 
${ }^{5}$ Utilizamos el plan de Minissa, posteriormente desarrollado por E. Roskam y J. Lingoes (versión de Edinburgh). Con la ayuda de un espacio tridimensional podemos describir perfectamente los datos de ambos grupos. En los grupos de nivel educativo más alto el énfasis llega a 0,1 ; en los de nivel más bajo, al 0,9 . El coeficiente de desviación de los grupos de menor nivel de educación es de 0,14 , $y$ el de los de mayor nivel, de 0,12 .

\section{GRAFICO I}

Configuración final para los grupos de bajo nivel educativo

\begin{tabular}{|c|c|c|c|}
\hline & 1 & 2 & 3 \\
\hline $\begin{array}{lllllllllll}1 & \ldots & \ldots & \ldots & \ldots & \ldots & \ldots & \ldots & \ldots & \ldots & \ldots\end{array}$ & $-1,1539$ & 0,1532 & $-0,1907$ \\
\hline $\begin{array}{llllllllllll} & \cdots & \ldots & \ldots & \ldots & \ldots & \ldots & \cdots & \ldots & \cdots & \cdots \\
2 & \ldots & \ldots & \ldots & \ldots & \ldots & \ldots & \ldots & \ldots & \ldots & \ldots\end{array}$ & 0,3639 & $-0,7114$ & 0,5872 \\
\hline 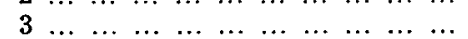 & 0,6195 & 0,8969 & 0,0802 \\
\hline $\begin{array}{lllllllllll}4 & \ldots & \ldots & \ldots & \ldots & \ldots & \ldots & \ldots & \ldots & \ldots & \ldots\end{array}$ & 0,4195 & $-0,8207$ & $-0,2244$ \\
\hline $\begin{array}{llllllllllll}5 & \ldots & \ldots & \ldots & \ldots & \ldots & \ldots & \ldots & \ldots & \ldots & \ldots\end{array}$ & 0,7402 & 0,1945 & $-0,3254$ \\
\hline $\begin{array}{llllllllllll}6 & \ldots & \ldots & \ldots & \ldots & \ldots & \ldots & \ldots & \ldots & \ldots & \ldots\end{array}$ & $-0,7983$ & $-0,6821$ & $-0,1984$ \\
\hline 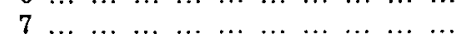 & 1,0318 & $-0,1325$ & $-0,1742$ \\
\hline 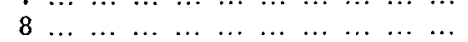 & 0,1834 & $-0,2480$ & 0,6975 \\
\hline $\begin{array}{lllllllllll}9 & \ldots & \ldots & \ldots & \ldots & \ldots & \ldots & \ldots & \ldots & \ldots & \ldots\end{array}$ & $-1,0895$ & 0,0209 & 0,1754 \\
\hline $\begin{array}{lllllllllll}10 & \ldots & \ldots & \ldots & \ldots & \ldots & \ldots & \ldots & \ldots & \ldots & \ldots\end{array}$ & $-0,1567$ & 0,5672 & 0,7453 \\
\hline $\begin{array}{lllllllllll}11 & \ldots & \ldots & \ldots & \ldots & \ldots & \ldots & \ldots & \ldots & \ldots & \ldots\end{array}$ & 0,0077 & $-0,2392$ & $-0,8195$ \\
\hline 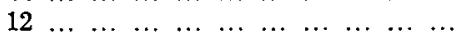 & $-0,1677$ & 1,0008 & $-0,3532$ \\
\hline $\begin{array}{lllllllll} & \\
\text { Media } & \ldots & \ldots & \ldots & \ldots & \ldots & \ldots & \ldots & \ldots\end{array}$ & $-0,0000$ & $-0,0000$ & $-0,0000$ \\
\hline $\begin{array}{lllllll} & \text { Desviación típica } & \ldots & \ldots & \ldots & \ldots & \ldots\end{array}$ & 8,6799 & 0,5752 & 0,4548 \\
\hline
\end{tabular}

\section{GRAFICO II}

Configuración final para los grupos de alto nivel educativo

\begin{tabular}{|c|c|c|c|}
\hline & 1 & 2 & 3 \\
\hline $\begin{array}{lllllllllll}1 & \ldots & \ldots & \ldots & \ldots & \ldots & \ldots & \ldots & \ldots & \ldots & \ldots\end{array}$ & $-0,4908$ & $-0,8355$ & 0,0259 \\
\hline $\begin{array}{lllllllllll}2 & \cdots & \ldots & \ldots & \ldots & \ldots & \ldots & \ldots & \ldots & \ldots & \ldots \\
2 & \ldots & \ldots & \ldots & \ldots & \ldots & \ldots & \ldots & \ldots & \ldots & \ldots\end{array}$ & 1,0075 & $-0,1742$ & $-0,1227$ \\
\hline $\begin{array}{llllllllllll} & \ldots & \ldots & \ldots & \ldots & \ldots & \ldots & \ldots & \ldots & \ldots & \ldots & \ldots\end{array}$ & $-0,3265$ & 0,8851 & 0,3297 \\
\hline $\begin{array}{lllllllllll}4 & \ldots & \ldots & \ldots & \ldots & \ldots & \ldots & \ldots & \ldots & \ldots & \ldots \\
4 & \ldots & \ldots & \ldots & \ldots & \ldots & \ldots & \ldots & \ldots & \ldots\end{array}$ & 1,0086 & $-0,1314$ & 0,1898 \\
\hline $\begin{array}{llllllllllll}5 & \ldots & \ldots & \ldots & \ldots & \ldots & \ldots & \ldots & \ldots & \ldots & \ldots\end{array}$ & 0,3345 & 0,6431 & $-0,5513$ \\
\hline $\begin{array}{llllllllllll}6 & \ldots & \ldots & \ldots & \ldots & \ldots & \ldots & \ldots & \ldots & \ldots & \ldots\end{array}$ & 0,1111 & $-0,5476$ & 0,7193 \\
\hline $\begin{array}{llllllllllll}7 & \ldots & \ldots & \ldots & \ldots & \ldots & \ldots & \ldots & \ldots & \ldots & \ldots\end{array}$ & 0,7182 & 0,6477 & 0,3751 \\
\hline $\begin{array}{llllllllllll}8 & 8 & \ldots & \ldots & \ldots & \ldots & \ldots & \ldots & \ldots & \ldots & \ldots & \ldots\end{array}$ & 0,4091 & $-0,6864$ & $-0,5892$ \\
\hline $\begin{array}{lllllllllll}9 & \ldots & \ldots & \ldots & \ldots & \ldots & \ldots & \ldots & \ldots & \ldots & \ldots\end{array}$ & $-0,7698$ & $-0,5461$ & 0,0798 \\
\hline $\begin{array}{cccccccccc}10 & \ldots & \ldots & \ldots & \ldots & \ldots & \ldots & \ldots & \ldots & \ldots\end{array}$ & $-0,6519$ & 0,1915 & 0,8163 \\
\hline $\begin{array}{lllllllllll}11 & \ldots & \ldots & \ldots & \ldots & \ldots & \ldots & \ldots & \ldots & \ldots & \ldots\end{array}$ & $-0,4041$ & 0,1383 & $-0,8887$ \\
\hline $\begin{array}{lllllllllll}12 & \ldots & \ldots & \ldots & \ldots & \ldots & \ldots & \ldots & \ldots & \ldots & \ldots\end{array}$ & $-0,9458$ & 0,4112 & $-0,3841$ \\
\hline $\begin{array}{llllllll}\text { Media } & \ldots & \ldots & \ldots & \ldots & \ldots & \ldots & \ldots\end{array} \ldots$ & $-0,0000$ & $-0,0000$ & $-0,0000$ \\
\hline Desviación típica $\ldots \ldots \ldots \ldots$ & 0,6622 & 0,5521 & 0,5066 \\
\hline
\end{tabular}


7 Aquí también es conveniente una descripción tridimensional. El factor énfasis es de $0,11, y$ el coeficiente de desviación, de 0,12.

\section{GRAFICO III}

\section{Configuración final}

\begin{tabular}{|c|c|c|c|}
\hline & 1 & 2 & 3 \\
\hline $\begin{array}{lllllllllll}1 & \ldots & \ldots & \ldots & \ldots & \ldots & \ldots & \ldots & \ldots & \ldots & \ldots\end{array}$ & $-0,2707$ & $-1,1252$ & 0,1044 \\
\hline $\begin{array}{lllllllllll}2 & \ldots & \ldots & \ldots & \ldots & \ldots & \ldots & \ldots & \ldots & \ldots & \ldots\end{array}$ & $-1,3415$ & 0,1008 & $-0,0601$ \\
\hline $\begin{array}{lllllllllll} & 3 & \ldots & \ldots & \ldots & \ldots & \ldots & \ldots & \ldots & \ldots & \ldots\end{array}$ & $-1,4329$ & 0,1957 & $-0,2740$ \\
\hline $\begin{array}{lllllllllll}4 & \ldots & \ldots & \ldots & \ldots & \ldots & \ldots & \ldots & \ldots & \ldots & \ldots\end{array}$ & $-1,2984$ & $-0,3443$ & $-0,2249$ \\
\hline $\begin{array}{lllllllllll}\mathbf{5} & \ldots & \ldots & \ldots & \ldots & \ldots & \ldots & \ldots & \ldots & \ldots & \ldots\end{array}$ & $-1,3266$ & $-0,3382$ & $-0,1747$ \\
\hline $\begin{array}{lllllllllll}6 & \ldots & \ldots & \ldots & \ldots & \ldots & \ldots & \ldots & \ldots & \ldots & \ldots\end{array}$ & $-1,0675$ & $-0,1954$ & $-0,5830$ \\
\hline $\begin{array}{lllllllllll}7 & \ldots & \ldots & \ldots & \ldots & \ldots & \ldots & \ldots & \ldots & \ldots & \ldots\end{array}$ & 0,8616 & 0,4842 & $-0,2784$ \\
\hline $\begin{array}{lllllllllll}8 & \ldots & \ldots & \ldots & \ldots & \ldots & \ldots & \ldots & \ldots & \ldots & \ldots\end{array}$ & 0,8258 & 0,6400 & $-0,4052$ \\
\hline $\begin{array}{lllllllllll}9 & \ldots & \ldots & \ldots & \ldots & \ldots & \ldots & \ldots & \ldots & \ldots & \ldots\end{array}$ & 1,0458 & 0,1622 & $-0,1312$ \\
\hline $\begin{array}{lllllllllll}10 & \ldots & \ldots & \ldots & \ldots & \ldots & \ldots & \ldots & \ldots & \ldots & \ldots\end{array}$ & 1,1302 & 0,0092 & $-0,2461$ \\
\hline $\begin{array}{lllllllllll}11 & \ldots & \ldots & \ldots & \ldots & \ldots & \ldots & \ldots & \ldots & \ldots & \ldots\end{array}$ & 1,1208 & 0,0996 & $-0,4227$ \\
\hline $\begin{array}{lllllllllll}12 & \ldots & \ldots & \ldots & \ldots & \ldots & \ldots & \ldots & \ldots & \ldots & \ldots\end{array}$ & $-0,1031$ & 0,0634 & $-0,9240$ \\
\hline $\begin{array}{lllllllllll}13 & \ldots & \ldots & \ldots & \ldots & \ldots & \ldots & \ldots & \ldots & \ldots & \ldots\end{array}$ & 0,3086 & $-0,2953$ & $-0,6927$ \\
\hline $\begin{array}{lllllllllll}14 & \ldots & \ldots & \ldots & \ldots & \ldots & \ldots & \ldots & \ldots & \ldots & \ldots\end{array}$ & $-0,2954$ & $-1,0420$ & $-0,1793$ \\
\hline $\begin{array}{lllllllllll}15 & \ldots & \ldots & \ldots & \ldots & \ldots & \ldots & \ldots & \ldots & \ldots & \ldots\end{array}$ & $-0,0539$ & 1,1469 & $-0,3130$ \\
\hline $\begin{array}{lllllllllll}16 & \ldots & \ldots & \ldots & \ldots & \ldots & \ldots & \ldots & \ldots & \ldots & \ldots\end{array}$ & 0,2022 & $-0,8954$ & $-0,6485$ \\
\hline $\begin{array}{lllllllllll}17 & \ldots & \ldots & \ldots & \ldots & \ldots & \ldots & \ldots & \ldots & \ldots & \ldots\end{array}$ & 0,3550 & $-0,0966$ & 0,5985 \\
\hline $\begin{array}{lllllllllll}18 & \ldots & \ldots & \ldots & \ldots & \ldots & \ldots & \ldots & \ldots & \ldots & \ldots\end{array}$ & $-0,5610$ & 0,4895 & 0,4569 \\
\hline $\begin{array}{lllllllllll}19 & \ldots & \ldots & \ldots & \ldots & \ldots & \ldots & \ldots & \ldots & \ldots & \ldots\end{array}$ & $-0,5921$ & 0,9094 & 0,0921 \\
\hline $\begin{array}{lllllllllll}20 & \ldots & \ldots & \ldots & \ldots & \ldots & \ldots & \ldots & \ldots & \ldots & \ldots\end{array}$ & 0,2573 & $-0,5503$ & 0,1780 \\
\hline $\begin{array}{lllllllllll}21 & \ldots & \ldots & \ldots & \ldots & \ldots & \ldots & \ldots & \ldots & \ldots & \ldots\end{array}$ & 0,3388 & 0,3880 & 0,4053 \\
\hline $\begin{array}{lllllllllll}22 & \ldots & \ldots & \ldots & \ldots & \ldots & \ldots & \ldots & \ldots & \ldots & \ldots\end{array}$ & 0,4203 & 0,0445 & 0,3322 \\
\hline $\begin{array}{lllllllllll}23 & \ldots & \ldots & \ldots & \ldots & \ldots & \ldots & \ldots & \ldots & \ldots & \ldots\end{array}$ & 0,1367 & 0,9953 & 0,3590 \\
\hline $\begin{array}{lllllllllll}24 & \ldots & \ldots & \ldots & \ldots & \ldots & \ldots & \ldots & \ldots & \ldots & \ldots\end{array}$ & $-0,5647$ & $-0,4554$ & $-0,0557$ \\
\hline $\begin{array}{lllllllllll}25 & \ldots & \ldots & \ldots & \ldots & \ldots & \ldots & \ldots & \ldots & \ldots & \ldots\end{array}$ & 0,5686 & $-0,4075$ & 0,2320 \\
\hline $\begin{array}{lllllllllll}26 & \ldots & \ldots & \ldots & \ldots & \ldots & \ldots & \ldots & \ldots & \ldots & \ldots\end{array}$ & $-0,0055$ & $-0,4524$ & 0,4265 \\
\hline $\begin{array}{lllllllllll}27 & \ldots & \ldots & \ldots & \ldots & \ldots & \ldots & \ldots & \ldots & \ldots & \ldots\end{array}$ & $-0,3386$ & $-0,0865$ & 0,4945 \\
\hline $\begin{array}{lllllllllll}28 & \ldots & \ldots & \ldots & \ldots & \ldots & \ldots & \ldots & \ldots & \ldots & \ldots\end{array}$ & $-0,4799$ & 0,9426 & 0,3755 \\
\hline $\begin{array}{lllllllllll}29 & \ldots & \ldots & \ldots & \ldots & \ldots & \ldots & \ldots & \ldots & \ldots & \ldots\end{array}$ & 0,1009 & $-0,5529$ & 0,3232 \\
\hline $\begin{array}{lllllllllll}30 & \ldots & \ldots & \ldots & \ldots & \ldots & \ldots & \ldots & \ldots & \ldots & \ldots\end{array}$ & 0,1475 & 0,3826 & 0,6899 \\
\hline $\begin{array}{lllllllllll}31 & \ldots & \ldots & \ldots & \ldots & \ldots & \ldots & \ldots & \ldots & \ldots & \ldots\end{array}$ & 0,2191 & $-0,1496$ & 0,4225 \\
\hline $\begin{array}{lllllllllll}32 & \ldots & \ldots & \ldots & \ldots & \ldots & \ldots & \ldots & \ldots & \ldots & \ldots\end{array}$ & $-0,0425$ & 0,8668 & 0,4827 \\
\hline $\begin{array}{llllllllllll}33 & \ldots & \ldots & \ldots & \ldots & \ldots & \ldots & \ldots & \ldots & \ldots & \ldots\end{array}$ & 0,5046 & $-0,4866$ & 0,0480 \\
\hline $\begin{array}{lllllllllll}34 & \ldots & \ldots & \ldots & \ldots & \ldots & \ldots & \ldots & \ldots & \ldots & \ldots\end{array}$ & 0,1049 & $-0,4644$ & 0,3953 \\
\hline $\begin{array}{lllllllllll}35 & \ldots & \ldots & \ldots & \ldots & \ldots & \ldots & \ldots & \ldots & \ldots & \ldots\end{array}$ & 0,2077 & $-0,8980$ & 0,2187 \\
\hline $\begin{array}{lllllllllll}36 & \ldots & \ldots & \ldots & \ldots & \ldots & \ldots & \ldots & \ldots & \ldots & \ldots\end{array}$ & 0,0838 & 0,9156 & $-1,0215$ \\
\hline 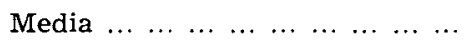 & $-0,0000$ & $-0,0000$ & $-0,0000$ \\
\hline $\begin{array}{llllll}\text { Desviación típica } & \ldots & \ldots & \ldots & \ldots & \ldots\end{array}$ & 0,6740 & 0,5981 & 0,4336 \\
\hline
\end{tabular}




\section{REFERENCIAS BIBLIOGRAFICAS}

Allard, E., y cols.: Questionnaire and Code Book of the Scandinavian Welfare Survey, 1972; Informe del Research Group for Corporative Sociology, Universidad de Helsinki, 14, 1977.

ANDREWs, F., y St. WitheY: Social Indicators of Well-Being, Nueva York, Londres, 1976.

BallerstedT, E., y W. Glazer: "Soziologischer Almanach, Handbunch gesellschaftlicher", en Daten für die Bundesrepublik Deutschland, Francfort, N. Y., 1975.

BARGE, T.: Zur Erfassung von Werten und Wertindikatoren im Projekt "Hochschulsozialisation", en Gesellschaft für Zukunftsfragen e.V./Gesamthochschule Paderborn, Werteinstellung und Wertwandel, Berlín/Paderborn (mimeo), 53 ff. 1978.

BELL, D.: The Coming of Postindustrial Society, Cambridge-Mass., 1973; The Cultural Contradictions of Capitalism, Nueva York, 1976.

Berger, P., y Th. Luckmann: The Social Construction of Reality, Nueva York, 1966.

Bronfenbrenner, U.: "Socialization through Time and Space", en Maccoby y cols. (eds.), Readings in Social Psychology, Nueva York, 1958, $400 \mathrm{ff}$.

DeUtsch, K.: "Social Mobilization and Political Development", en Am. P.S.R., 55, 1961,498 ff.

Etzion, A.: The Active Society, Londres, Nueva York, 1968.

Gutrman, L.: "A General Nonmetric Technique for Finding the Smallest Space for a Configuration of Points", en Psychometrika, 1968, $469 \mathrm{ff}$.; "Social Problem Indicators", en The Annals of the American Academy of Political and Social Science, 393, 1971, $40 \mathrm{ff}$.

- y SH. Levy: "Values and Attitudes of Israel High School Youth, 2nd", en Research Project, The Israel Institute of Applied Social Research, Jerusalén (mimeo), 1976.

HabmRas, J.: "Stichwerte zu einer Theorie der Sozialisation", en Habermas, Kultur und Kritik, Francfort, 1973, 118 ff.

HaRRIEs-Jenkins, G., y J. vaN Doorn (eds.): The Military and the Problem of Legitimacy, Beverly Hills, 1976.

Hernz, W.: "Gesellschaftsstruktur, familiale Sozialisation und gesellschaftliches Bewusstsein", en Hurrelmann, 1976, $90 \mathrm{ff}$.

HurrelmanN, R. (ed.): Sozialisation und Lebenslauf, Reinbak, 1976.

Inglemart, R.: The Silent Revolution, Princeton, 1977.

IRLE, M.: Lehrbuch der Socialpsychologie, Göttinger, 1975.

KLAGES, H.: Die unruhige Gesellschaft, Munich, 1975.

KLAPPER, J. T.: The Effects of Mass Comunication, Glencoe, 1960.

KMIsciak, P.: Wertstrukturen und Wertwandel in der Bundesrepublik Deuthland, Berlín, 1976, 316 ff.

KoHN, M. L.: Class and Conformity, Homewood, IIl., 1969.

LERNER, D.: The Passing of Traditional Society: Modernizing the Middle East, 2. ${ }^{\text {a }}$ ed., Nueva York, 1964, (58).

LIPSET, S. M., y R. B. Dosson: "The Intellectual as Critic and Rebel", en Daedalus, $10,1972,137$ ff.

LÜSCHER, K.: "Perspektiven einer Soziologie der Sozialisation", en Zeitschrift für Soziologie, 4, 1975, $359 \mathrm{ff}$.

Marsh, A.: Protest and Political Consciousness, Beverly Hills, 1977.

Noelle-Neumann, E.: "Werder wir alle Proletarier?", en Die Zeit, 25-26, 1976.

RübGG, W.: Anstösse, Frankfurt-Main, 1973.

- Bedrohte lebensordnung, Zürich, 1978.

Rescher, N.: "What is Value Change? A Framework for Research", en K. Baier y N. Rescher (eds.), Values and the Future, Nueva York, 1969.

Runcrman, W. G.: Relative Deprivation and Social Justice, Londres, 1968.

Werss, K.: "Mass Media and Social Change", en King-McGinnies (eds.), Attitudes, Conflict and Social Change, Nueva York, 1972, $175 \mathrm{ff}$.

Wright, J. D., y S. R. Wright: "Social class and Parental Values for Children", en $A S R, 41,1976,527$ ff. 